\title{
Magnitude of undernutrition in children aged 2 to 4 years using CIAF and conventional indices in the slums of Mumbai city
}

\author{
Mitravinda S. Savanur *D and Padmini S. Ghugre
}

\begin{abstract}
Conventional indicators - weight-for-age, height-for-age, weight-for-height and mid-upper arm circumference (MUAC) reflect different facets of the nutritional status. Weight-for-age is the most commonly used indicator. When used individually or in combination, conventional indices fail to depict the overall magnitude of undernutrition in the population. Composite Index of Anthropometric Failure (CIAF) is an alternative classification system which attempts to fill this lacuna. Thus, we undertook this study with the objective to compare the prevalence of undernutrition using CIAF and the conventional indices. We included 634 children aged between 2 to 4 years from anganwadis located in three areas of Mumbai. Weight, height and MUAC measurements were taken. Z scores were computed for weight-for-age (WAZ), height-for-age (HAZ) and weight-for-height (WHZ) using WHO Anthro software. Children were classified as per the conventional indices and CIAF. The prevalence of underweight, stunting and wasting was $35.7 \%, 33.8 \%$ and $18.5 \%$ respectively. None of the children had MUAC $<11.5 \mathrm{~cm}$. About $1 \%$ of the children were moderately wasted according to MUAC. As per CIAF, $47.8 \%$ children were undernourished. According to $\mathrm{CIAF}$, one-third of the undernourished children had single anthropometric failure while half of them had dual failure and $17.1 \%$ had multiple failures. When compared with the conventional indices, CIAF could recognize $12.1 \%, 14.0 \%, 29.3 \%$ and $46.7 \%$ more undernourished children than WAZ, HAZ, WHZ and MUAC respectively. In conclusion, CIAF is seen to have many advantages over the conventional indices. CIAF is useful in assessing the overall magnitude of undernutrition and identifying children with multiple anthropometric failures. It also recognizes more undernourished children than all the conventional indices. Therefore, CIAF should be used more widely as a tool for nutritional assessment particularly in developing countries where the burden of undernutrition is high.
\end{abstract}

Keywords: CIAF, Underweight, Stunting, Wasting, Nutritional status, Children, India

\section{Background}

Undernutrition among children under five years is traditionally assessed using anthropometric indices such as - weight-for-age, height-for-age, weight-for-height and mid-upper arm circumference (MUAC). Stunting or low height-for-age is an indicator of chronic undernutrition which is manifested as poor skeletal growth. Low weightfor-height reflects wasting or acute undernutrition with loss of lean as well as fat mass [1]. On the other hand, low MUAC $(<11.5 \mathrm{~cm})$ is not only suggestive of severe wasting or severe acute malnutrition but also indicative of morbidity and risk of mortality [2, 3]. Underweight or low

\footnotetext{
* Correspondence: mitrasav@gmail.com

Department of Food Science and Nutrition, Sir Vithaldas Vihar, S.N.D.T. Women's University, Juhu Road, Mumbai 400049, Maharashtra, India
}

weight-for-age, on the other hand, is indicative of both acute and chronic undernutrition [1]. These indices reflect different facets of undernutrition. Although underweight, stunting and wasting reflect different facets of undernutrition, they are not mutually exclusive categories. For instance, a child who is found to be stunted can also be underweight and wasted at the same time. Hence, a sum of the children who are underweight, stunted and wasted in a group does not reveal the overall number of undernourished children in a population. The conventional indices therefore fail to provide the overall prevalence of undernutrition in a group.

There is another concern with the use of conventional indices. Weight-for-age is most commonly used to assess 
the nutritional status. This may be because underweight indicates both acute and chronic undernutrition. However, underweight is not the summation of children who are wasted and stunted. As a result, we might tend to miss out on children who are stunted and wasted if underweight is used as a sole indicator of nutritional status.

In the year 2000, Swedish Economist Prof. Peter Svedberg suggested an alternative measure to assess the overall magnitude of undernutrition - Composite Index of Anthropometric Failure (CIAF). CIAF identifies seven groups of children including those without any form of anthropometric failure (Table 1). A summation of the groups B, C, D, E, F and Y gives the total magnitude of undernutrition. At the same time, it can be useful in detecting multiple anthropometric failures [4].

Investigators in Kenya, China and Bangladesh have used CIAF to assess the extent of undernutrition [5-7]. In Kenya, Berger et al estimated the prevalence of undernutrition among children with HIV/AIDS [5]. Khan et al and Pei et al also identified the sociodemographic factors determining overall undernutrition $[7,6]$. Nandy and Miranda have used the national data from seven developing countries to calculate the CIAF and compare it with prevalence of underweight in the same areas [8].

In India, Nandy et al was the first to use the concept of CIAF on the data of 1998 - 99 National Family Health Survey - 2 (NFHS - 2) [1]. Thereafter most of the Indian studies that have used CIAF have been conducted in rural or tribal areas of West Bengal [9-15]. Only two studies so far have used CIAF in an urban setting i.e. in Coimbatore, Tamil Nadu and Bankura town, West Bengal [16, 17].

In Maharashtra state, the prevalence of underweight, stunting and wasting in under-five children in rural and urban areas was $37 \%, 46 \%$ and $17 \%$ respectively. The nutritional status of under-five children in Mumbai was

Table 1 Categories of the composite index of anthropometric failure (CIAF)

\begin{tabular}{|c|c|c|}
\hline Group & Description of the group & Definition \\
\hline A & No anthropometric failure & Normal WAZ, HAZ and WHZ \\
\hline B & Wasting only & $\begin{array}{l}\text { WHZ }<-2 S D \text { but normal WAZ } \\
\text { and } \mathrm{HAZ}\end{array}$ \\
\hline C & Wasting and underweight & $\begin{array}{l}\text { WHZ and } W A Z<-2 \text { SD but } \\
\text { normal HAZ }\end{array}$ \\
\hline D & $\begin{array}{l}\text { Wasting, underweight and } \\
\text { stunting }\end{array}$ & WHZ, WAZ and $H A Z<-2$ SD \\
\hline $\mathrm{E}$ & Stunting and underweight & $\begin{array}{l}\text { HAZ and WAZ }<-2 \text { SD but } \\
\text { normal } W H Z\end{array}$ \\
\hline $\mathrm{F}$ & Stunting only & $\begin{array}{l}H A Z<-2 \text { SD but normal WAZ } \\
\text { and } W H Z\end{array}$ \\
\hline Y & Underweight only & $\begin{array}{l}\text { WAZ }<-2 \text { SD but normal HAZ } \\
\text { and } W H Z\end{array}$ \\
\hline
\end{tabular}

reportedly worse than the other urban areas in the state. The prevalence of underweight and stunting was $40 \%$ and $14 \%$ higher respectively in the slum than the nonslum areas of Mumbai [18]. The overall extent of undernutrition in the children belonging to the slums in Mumbai remains unknown.

We therefore conducted the present study with an objective to compare the prevalence of undernutrition by using CIAF and the conventional indices.

\section{Methods}

The study was approved by Independent Ethics Committee (IEC no 09122), Navi Mumbai, Maharashtra, India.

\section{Study design}

This cross-sectional study was carried out from July, 2013 to January, 2014 in the slums of Mumbai city, Maharashtra, India. We undertook the study in three urban slum areas located in the western suburbs of the city. Each of these areas had 140 to 150 anganwadis each. Anganwadi is a child-care and mother-care centre which is run by the Integrated Child Development Service (ICDS) in India. We obtained a list of anganwadis in each of these areas from the respective Child Development Project Officers (CDPO). From this list, every sixth anganwadi was selected by simple random sampling. Thus, from every area, we selected 25 anganwadis each (Fig. 1). Participants: Six hundred and thirty four children aged between 2 to 4 years participated in the study. The inclusion and exclusion criteria for including the children in this study were.

\section{Inclusion criteria}

Children who (i) were beneficiaries of the anganwadi, (ii) had authentic records of their date of birth and (iii) had completed 24 months of age and were less than or equal to 48 months were included in the study. Age was calculated on the basis of their date of birth to the nearest one month.

\section{Exclusion criteria}

Children who were - (i) suffering from any chronic illness that influenced their nutritional status, (ii) born with congenital anomalies, (iii) born extremely premature ( $<28$ weeks of gestational age).

Parents and/or guardians of the children were explained about the study procedure in the local language and an informed consent was obtained from them.

\section{Assessment of nutritional status}

Weight and height measurements of all the children were taken using standard procedure [19]. Children were weighed on a digital weighing scale (Dr Gene Health and Wellness; Model no: MS8270) with an accuracy of $0.1 \mathrm{~kg}$. Height was measured using a non- 


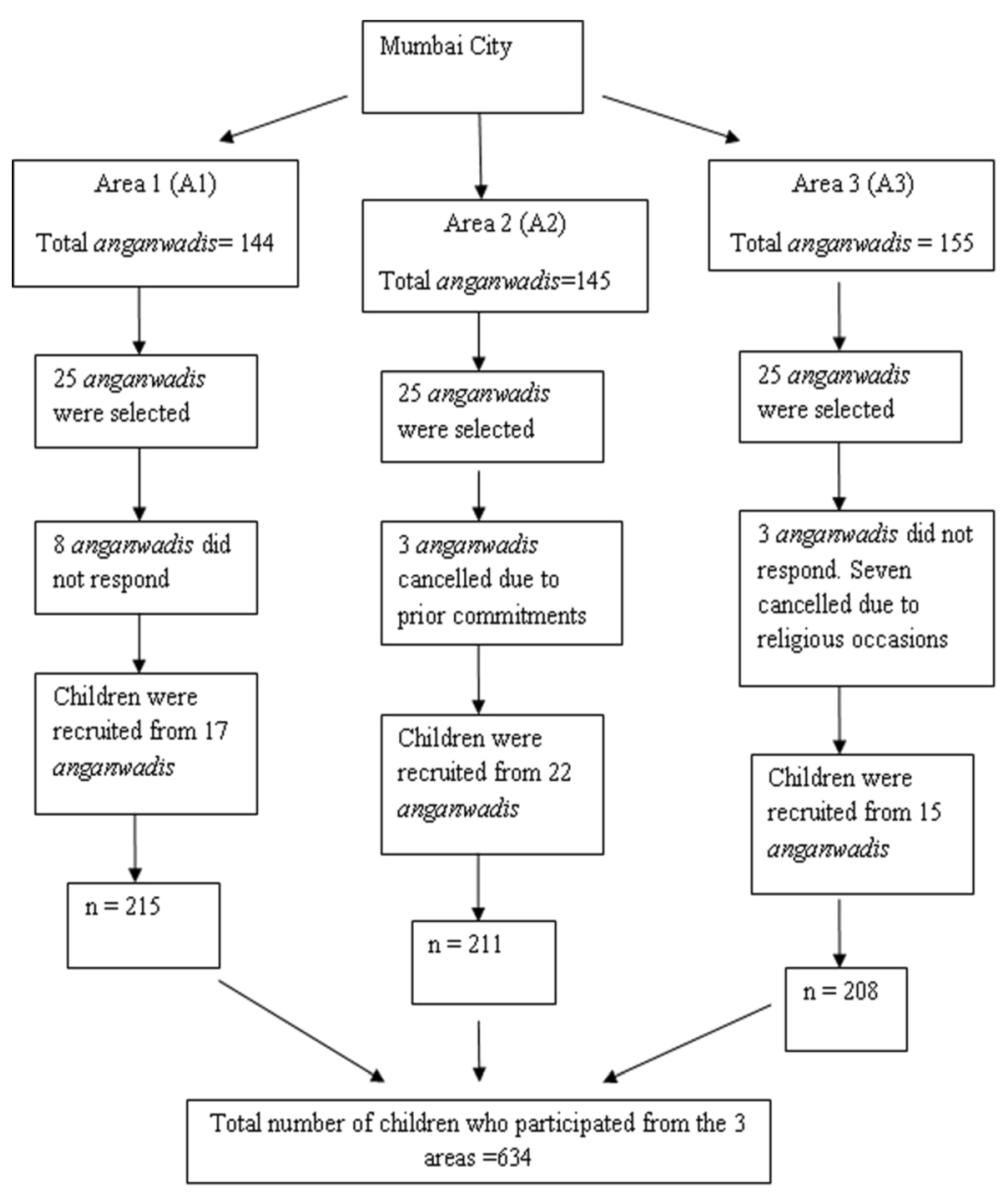

Fig. 1 Selection of Participants from Mumbai City

extensible, flexible measuring tape with an accuracy of $0.1 \mathrm{~cm}$ which was calibrated against the standard anthropometric scale. Based on these measurements, weight-for-age $\mathrm{Z}$ scores (WAZ), height-for-age $\mathrm{Z}$ scores (HAZ) and weight-for-height Z scores (WHZ) were computed. MUAC was measured using a nonextensible, flexible measuring tape at the mid-point between the acromion and olecranon processes of the child's arm flexed at $90^{\circ}$ angle. The child was then made to relax the arm so that it hangs just away from the side of the body and the circumference was measured at the mid-point with an accuracy of $0.1 \mathrm{~cm}$ [20].

Children with WAZ, HAZ and WHZ scores between 3.0 to $-2.0 \mathrm{SD}$ were classified as moderately underweight, stunted and wasted respectively. Those with WAZ, HAZ and WHZ scores were $<-3.0$ SD were classified as severely underweight, stunted and wasted respectively. And, those children who had Z scores $>-2.0 \mathrm{SD}$ were classified as 'normal' [21]. MUAC $<11.5 \mathrm{~cm}$ and between 11.5 to $12.5 \mathrm{~cm}$ were classified as severe acute malnutrition (SAM) and moderate acute malnutrition (MAM) respectively. Those with MUAC $>12.5 \mathrm{~cm}$ were considered 'normal' [3]. Further, children were also classified using CIAF [1].

\section{Statistical analysis}

WAZ, HAZ and WHZ scores were computed using WHO Anthro software version 3.2.2. The data was analyzed using SPSS version 20. For WAZ, HAZ, WHZ and MUAC one-way ANOVA was used to analyze the difference in the mean $\mathrm{Z}$ scores between boys and girls. Chisquare tests were performed to study the age-wise and gender-wise differences in the prevalence of undernutrition.

\section{Results}

There were $53.6 \%(n=340)$ boys and $46.4 \%(n=294)$ girls in the study. The mean age of the children was $3.1 \pm 0.8$ years. The mean anthropometric measurements of children are presented in Table 2. Boys weighed significantly more $(\mathrm{F}=12.039, \mathrm{p}=0.001)$ and had a significantly higher MUAC $(\mathrm{F}=4.151, \mathrm{p}=0.042)$ than the girls. 
Table 2 Mean anthropometric measurements of boys and girls $(n=634)$

\begin{tabular}{lcccc}
\hline Parameter & Boys $(n=340)$ & Girls $(n=294)$ & $F$ & $P$ value \\
\hline Weight $(\mathrm{kg})$ & $11.9 \pm 1.8$ & $11.4 \pm 1.8$ & 12.039 & 0.001 \\
Height $(\mathrm{cm})$ & $90.1 \pm 7.2$ & $89.2 \pm 7.0$ & 2.427 & 0.120 \\
WAZ & $-1.6 \pm 1.0$ & $-1.7 \pm 0.9$ & 2.194 & 0.139 \\
HAZ & $-1.5 \pm 1.2$ & $-1.6 \pm 1.1$ & 0.921 & 0.337 \\
WHZ & $-1.1 \pm 1.0$ & $-1.1 \pm 0.9$ & 0.000 & 0.987 \\
MUAC $(\mathrm{cm})$ & $14.9 \pm 1.1$ & $14.8 \pm 1.1$ & 4.151 & 0.042
\end{tabular}

WAZ Weight-for-age Z score, HAZ Height-for-age Z score, WHZ Weight-for-height $Z$ score, MUAC Mid-upper arm circumference

However no statistical differences were seen between boys and girls for the other anthropometric parameters.

\section{Nutritional Status}

\section{Using conventional indices}

Children were classified as per WHO Growth Standards $[3,21]$. One hundred and seventy-six $(27.8 \%)$ children were moderately underweight and 50 (7.9\%) were severely underweight (Table 3 ). One hundred and fortythree $(22.6 \%)$ children and 71(11.2 \%) were moderately and severely stunted respectively. One hundred and four (16.4\%) children and $13(2.1 \%)$ children had moderate and severe wasting respectively. None of the children had a MUAC $<11.5 \mathrm{~cm}$. Seven children (1.1 \%) had
MAM according to MUAC. There were no significant differences were observed in the prevalence rates in boys and girls. Similarly, no statistical differences were seen in the prevalence of underweight and stunting across the age groups. However, significantly higher number of children in the age group of 3 to 4 years were moderately wasted than children aged 2 to 3 years of age (20.3\% v $12 \% ; \not 2=7.983, \mathrm{p}=0.018)$.

\section{Using composite index of anthropometric failure}

According to CIAF classification, 331 children (52.2\%) were well nourished (Table 4). Nearly half the children i.e. $303(47.8 \%)$ were undernourished. Of all the undernourished children, 101 children (33.3\%) suffered from single anthropometric failure (Group - B, F and Y). Almost half the undernourished children (49.5\%) suffered from dual anthropometric failure (Group - C and E) while fifty-two children i.e. $17.1 \%$ experienced multiple failures i.e. they were underweight, stunted and wasted at the same time. No age-wise $(\chi 2=11.516, \mathrm{p}=0.074)$ and sex-wise $(\chi 2=10.864, p=0.093)$ differences were noted in the prevalence of single, dual and multiple anthropometric failures.

\section{CIAF v Conventional indices of undernutrition}

CIAF could identify more undernourished children than the conventional indices. The prevalence of undernutrition

Table 3 Prevalence of undernutrition according to the conventional indices

\begin{tabular}{|c|c|c|c|c|c|c|}
\hline \multirow[t]{2}{*}{ Indicator } & \multirow[t]{2}{*}{ Classification } & \multicolumn{2}{|l|}{ Age (years) } & \multicolumn{2}{|l|}{ Sex } & \multirow{2}{*}{$\begin{array}{l}\text { Total \% } \\
\text { (n) }\end{array}$} \\
\hline & & 2 to $3 \%(n)$ & 3 to $4 \%(n)$ & Boys \% (n) & Girls \% (n) & \\
\hline \multirow[t]{5}{*}{ WAZ (Underweight) } & Normal & 64.5 (209) & 64.2 (199) & $66.8(227)$ & $61.6(181)$ & $64.4(408)$ \\
\hline & Moderate underweight & $29.6(96)$ & $25.8(80)$ & $25.6(87)$ & $30.3(89)$ & $27.8(176)$ \\
\hline & Severe underweight & $5.9(19)$ & $10.0(31)$ & $7.6(26)$ & $8.2(24)$ & $7.9(50)$ \\
\hline & $x^{2}$ & 4.273 & & 1.962 & & \\
\hline & $\mathrm{p}$ & 0.118 & & 0.375 & & \\
\hline \multirow[t]{5}{*}{ HAZ (Stunting) } & Normal & $66.0(214)$ & $66.5(206)$ & $67.6(230)$ & $64.6(190)$ & $66.2(420)$ \\
\hline & Moderate stunting & $22.2(72)$ & $22.9(71)$ & $20.9(71)$ & $24.5(72)$ & $22.6(143)$ \\
\hline & Severe stunting & $11.7(38)$ & $10.6(33)$ & $11.5(39)$ & $10.9(32)$ & $11.2(71)$ \\
\hline & $x^{2}$ & 0.202 & & 1.175 & & \\
\hline & $\mathrm{p}$ & 0.904 & & 0.556 & & \\
\hline \multirow[t]{5}{*}{ WHZ (Wasting) } & Normal & $85.8(278)$ & $77.1(239)$ & $79.7(271)$ & $83.7(246)$ & $81.5(517)$ \\
\hline & Moderate wasting & $12.7(41)$ & $20.3(63)$ & $17.9(61)$ & $14.6(43)$ & $16.4(104)$ \\
\hline & Severe wasting & $1.5(5)$ & $2.6(8)$ & $2.4(8)$ & $1.7(5)$ & $2.1(13)$ \\
\hline & $x^{2}$ & 7.983 & & 1.688 & & \\
\hline & $p$ & 0.018 & & 0.430 & & \\
\hline \multirow[t]{4}{*}{ MUAC (Acute malnutrition) } & Normal (>12.5cm) & $99.1(321)$ & $98.7(306)$ & $98.8(336)$ & $99.0(291)$ & $98.9(627)$ \\
\hline & MAM (11.5 to $12.5 \mathrm{~cm})$ & $0.9(3)$ & $1.3(4)$ & $1.2(4)$ & $1.0(3)$ & $1.1(7)$ \\
\hline & $x^{2}$ & 0.193 & & 0.035 & & \\
\hline & $p$ & 0.720 & & 1.000 & & \\
\hline
\end{tabular}


Table 4 Prevalence of undernutrition according to CIAF

\begin{tabular}{|c|c|c|c|c|c|c|}
\hline \multirow[t]{2}{*}{ Group } & \multirow[t]{2}{*}{ Description of the group } & \multicolumn{2}{|l|}{ Age (years) } & \multicolumn{2}{|l|}{ Sex } & \multirow{2}{*}{$\begin{array}{l}\text { Total \% } \\
\text { (n) }\end{array}$} \\
\hline & & 2 to $3 \%(n)$ & 3 to $4 \%(n)$ & Boys \% (n) & Girls \% (n) & \\
\hline $\bar{A}$ & No anthropometric failure & $53.7(174)$ & $50.6(157)$ & $54.1(184)$ & $50.0(147)$ & $52.2(331)$ \\
\hline B & Wasting only & $1.2(4)$ & $4.2(13)$ & $3.8(13)$ & $1.3(4)$ & $2.6(17)$ \\
\hline C & Wasting and underweight & $6.2(20)$ & $8.7(27)$ & $6.7(23)$ & $8.5(25)$ & $7.5(48)$ \\
\hline $\mathrm{D}$ & Wasting, underweight and stunting & $6.8(22)$ & $9.7(30)$ & $9.7(33)$ & $6.4(19)$ & $8.2(52)$ \\
\hline E & Stunting and underweight & $17.6(57)$ & $14.8(46)$ & $13.8(47)$ & $18.7(55)$ & $16.1(102)$ \\
\hline $\mathrm{F}$ & Stunting only & $9.6(31)$ & $9.4(29)$ & $8.8(30)$ & $10.2(30)$ & $9.4(60)$ \\
\hline \multirow[t]{4}{*}{ Y } & Underweight only & $4.9(16)$ & $2.6(8)$ & $2.9(10)$ & $4.76(14)$ & $3.7(24)$ \\
\hline & Total anthropometric failure $(B+C+D+E+F+Y)$ & $46.3(150)$ & $49.4(153)$ & $45.8(156)$ & $50.0(147)$ & $47.8(303)$ \\
\hline & $x^{2}$ & \multicolumn{2}{|l|}{11.516} & \multicolumn{2}{|l|}{10.864} & \\
\hline & $\mathrm{p}$ & \multicolumn{2}{|l|}{0.074} & \multicolumn{2}{|l|}{0.093} & \\
\hline
\end{tabular}

according to CIAF was $47.8 \%$. On the other hand, using conventional indices, $35.7 \%$ were underweight, $33.8 \%$ were stunted and $18.5 \%$ were wasted. Thus, CIAF could recognize $12.1 \%, 14.0 \%$ and $29.3 \%$ more undernourished children than WAZ, HAZ and WHZ respectively. CIAF could recognize $46.7 \%$ more children as undernourished than MUAC. Among all the children classified as well nourished by MUAC, $16.1 \%$ had single anthropometic failure while $23.4 \%$ and $7.7 \%$ suffered from dual and multiple failures respectively (Table 5).

\section{Discussion}

The present study compared the prevalence of undernutrition by using CIAF and the conventional indices. The prevalence of undernutrition according to CIAF was 47.8 $\%$. On the other hand, according to the conventional indices $35.7 \%$, $33.8 \%$ and $18.5 \%$ children were underweight, stunted and wasted respectively. Also, about $1 \%$ children were classified as MAM according to MUAC.

Some researchers have used CIAF to assess the prevalence of undernutrition. Nandy and Miranda [8] computed the CIAF using the national data from 1998 to 2001 of seven developing countries - India, Ethopia, Nepal,

Table 5 Distribution of children in MUAC categories across the CIAF

\begin{tabular}{lll}
\hline CIAF Classification & \multicolumn{2}{l}{ MUAC Categories \% $(\mathrm{n})$} \\
\hline & 11.5 to $12.5 \mathrm{~cm}$ & $>12.5 \mathrm{~cm}$ \\
& $(\mathrm{n}=7)$ & $(\mathrm{n}=627)$ \\
No failure & 0 & $52.8(329)$ \\
Wasting only & 0 & $2.7(17)$ \\
Wasting and underweight & $14.3(1)$ & $7.3(46)$ \\
Wasting, underweight and stunting & $57.1(4)$ & $7.7(48)$ \\
Stunting and underweight & $28.6(2)$ & $16.1(101)$ \\
Stunting only & 0 & $9.6(60)$ \\
Underweight only & 0 & $3.8(24)$ \\
\hline
\end{tabular}

Tanzania, Zimbabwe, Bolivia and Peru. The prevalence rates in Peru (23.3\%), Bolivia (26.6\%) and Zimbabwe (35.8\%) were lower than that observed our study while in Nepal (56.5 \%) and Ethopia (58 \%) rates were much higher. Recent reports indicate that $21.4 \%$ children were undernourished in rural China [6] while in rural Bangladesh the prevalence was $58.7 \%$ [7]. On the other hand, the rates were slightly lower in urban Bangladesh (47.9\%) and were comparable to that observed in our study [7].

In India, a total of $59.8 \%$ children were reported to be undernourished using the NFHS - 2 data collected during 1998 - 99 [8]. Other studies in India have been mainly conducted in rural and tribal West Bengal wherein the prevalence rates ranged from 50.2 to $73.0 \%$ which were much higher than those observed in our study [9-14]. Similarly, $59.6 \%$ were reported to be undernourished in rural Wardha, Maharashtra state [22]. Factors associated with undernutrition in these rural and tribal areas were reported to be birth order, low birth weight, breastfeeding practices and mother's education [15]. Only two studies have been reported from urban areas of India, one from Tamil Nadu [16] and another from West Bengal [17]. The prevalence rates in these studies were higher than our study. These differences can be possibly attributed to various factors such as socioeconomic status, educational level, living conditions, maternal health, birth weight, feeding practices and rates of infections. However, we have not studied the factors influencing the overall prevalence of undernutrition as a part of this paper.

In the studies where CIAF and conventional indices were used together, CIAF identified more undernourished children than the latter $[8,14,16,23]$. Nandy and Miranda [8] found that the use of weight-for-age underestimated the prevalence by 9.7 to $21.7 \%$ as compared to CIAF. Similar findings have been noted by some other researchers in India [14, 16, 23]. However, these workers 
compared CIAF with only WAZ. We compared CIAF with WAZ, HAZ, WHZ and MUAC. WAZ underestimated the prevalence by $12.1 \%$, HAZ by $14.0 \%$ and WHZ by $29.3 \%$ as compared to CIAF. Also, MUAC identified $1.1 \%$ children as MAM but failed to identify $46.7 \%$ children who had varying degrees of undernutrition. Thus the conventional indices underestimated the prevalence of undernutrition and erroneously classified a sizeable section of the population as 'normal'.

Further, conventional indicators do not give a holistic picture of the nutritional status of a population. For instance, by using conventional indices we can find out how many children are underweight. But, there could be some children who are wasted as well as underweight. Such groups of children suffering from two or more anthropometric failures remain unidentified when conventional measures are used. In view of this, Svedberg added three categories to the existing conventional indicators (Group C: wasting and underweight, D: underweight, stunting and wasting and $\mathrm{E}$ : underweight and stunting) [4]. In this study, only $33 \%$ of the undernourished children exhibited single anthropometric failure while the remaining had either dual or multiple anthropometric failures which was not revealed with the use of conventional indices. These children exhibiting multiple anthropometric failures will require regular counseling and monitoring than those having single anthopometric failure.

In India, ICDS is the national programme that aims at reducing undernutrition and improving the nutritional status of children up to six years of age. Presently, the ICDS uses weight-for-age and MUAC as the indicators of nutritional status. WAZ identifies underweight children while MUAC recognizes those with extreme wasting. Stunted and moderately/severely wasted children are not identified as undernourished. As a result, a significant proportion of children suffering from dual or multiple failures are missed out. These vulnerable children fail to receive adequate attention and appropriate counseling from the anganwadi workers. This can further increase the burden of undernutrition and risk for morbidity. Thus, with the use of CIAF all the undernourished children can be identified.

The ICDS routinely weighs the children. If height is also measured then CIAF can be easily used. Using CIAF will not incur any extra financial burden on the government machineries. The use of CIAF will not only help in assessment but also in monitoring the growth of these children suffering from various degrees of anthropometric failure. Parents can be sensitized and appropriately counseled regarding their child's nutritional status. Such steps can help reduce the overall burden of undernutrition and also pave the path towards attaining the Millennium Development Goals (MDGs).
The strength of the present study is that this is the first study to have used CIAF to estimate the overall prevalence of undernutrition in the slums of Mumbai. Also, the study compares the prevalence rates of CIAF with all the conventional indices including MUAC. The limitation of our study was that it was carried out in three slum areas of Mumbai city. It would have been worthwhile if similar data was gathered from the nonslum areas where people across the socioeconomic spectrum reside. Also, we used only anthropometric indices and did not include any biochemical parameters for the assessment of nutritional status.

\section{Conclusion}

CIAF is a useful tool which provides a holistic picture of the overall prevalence of undernutrition. Conventional indicators when used individually grossly underestimate the extent of undernutrition. ICDS should thus, consider using CIAF at the national level as a step towards reducing the overall burden of undernutrion in children.

\section{Competing interests}

The authors declare that they have no competing interests.

\section{Authors' contributions}

MSS and PSG conceptualized and designed the study. MSS collected and analyzed the data. MSS drafted the manuscript. PSG provided critical inputs to the manuscript. All authors read and approved the final manuscript.

\section{Acknowledgement}

We wish to acknowledge the continuous support and co-operation of the CDPOs, anganwadi workers and helpers.

Received: 29 May 2015 Accepted: 26 June 2015

Published online: 10 July 2015

\section{References}

1. Nandy S, Irving M, Gordon D, Subramaniun SV, Smith GD. Poverty, child undernutrition and morbidity: new evidence from India. Bull World Health Organ. 2005;83(3):210-6.

2. Berkley J, Mwangi I, Griffiths K, Ahmed I, Mithwani S, English M, et al. Assessment of severe malnutrition among hospitalized children in Rural Kenya: comparison of weight for height and mid upper arm circumference. J Am Med Assoc. 2005;294:591-7.

3. World Health Organization and UNICEF. WHO child growth standards and the identification of severe acute malnutrition in infants and children. A Joint Statement by the World Health Organization and the United Nations Children's Fund. Geneva: World Health Organization; 2009.

4. Svedberg P. Poverty and undernutrition: theory, measurement and policy. New Delhi: Oxford India Paperbacks; 2000.

5. Berger MR, Fields-Gardner C, Wagle A, Hollenbeck CB. Prevalence of malnutrition in human immunodeficiency virus/acquired immunodeficiency syndrome orphans in the Nyanza Province of Kenya: a comparison of conventional indexes with a composite index of anthropometric failure. J Am Diet Assoc. 2008;108:1014-7.

6. Pei L, Ren L, Yan H. A survey of undernutrition in children under three years of age in rural Western China. BMC Public Health. 2014;14:121-31.

7. Khan REA, Raza MA. Nutritional status of children in Bangladesh: measuring composite index of anthropometric failure (CIAF) and its determinants. Pak J Commer Soc Sci. 2014;8(1):11-23.

8. Nandy S, Miranda JJ. Overlooking undernutrition? Using composite index of anthropometric failure to assess how underweight misses and misleads the assessment of undernutrition in young children. Soc Sci Med. 2008;66(9-5):1963-6. 
9. Biswas S, Bose K, Mukhopadhyay A, Bhadra M. Prevalence of undernutrition among pre-school children of Chapra, Nadia District, West Bengal, India, measured by composite index of anthropometric failure (CIAF). Anthropol Anz. 2009;67(3):269-79.

10. Mandal GC, Bose K. Assessment of overall prevalence of undernutrition using composite index of anthropometric failure (CIAF) among preschool children of West Bengal, India. Iran J Pediatr. 2009;19(3):237-43.

11. Das $\mathrm{S}$, Bose K. Assessment of nutritional status by anthropometric indices in Santal Tribal Children. J Life Sci. 2011;3(2):81-5.

12. Sen J, Dey S, Mondal N. Conventional nutritional indices and Composite Index of Anthropometric Failure: which seems more appropriate for assessing under-nutrition among children? A cross-sectional study among school children of the Bengalee Muslim Population of North Bengal, India. Italian J Public Health. 2011;8(2):172-84.

13. Sinha NK, Maiti S. Prevalence Of Undernutrition Among Underprivileged Preschool Children (2-6 years) Of Midnapore Town, India Malaysian Journal Of Paediatrics And Child Health Online Early 2012; 18 (1). Available at http.// mjpch.com/index.php/mjpch/article/viewFile/201/202.

14. Acharya A, Mandal GC, Bose K. Overall burden of under-nutrition measured by a Composite Index in rural pre-school children in Purba Medinipur, West Bengal, India. Anthropol Rev. 2013;76(1):109-16.

15. Dasgupta A, Parthasarathi R, Prabhakar R, Biswas R, Geethanjali A. Assessment of under nutrition with composite index of anthropometric failure (CIAF) among under-five children in a Rural Area of West Bengal, Indian. J Community Health. 2014;26(2):132-8.

16. Seetharam N, Chacko TV, Shankar SLR, Mathew AC. Measuring malnutrition The role of Z-scores and composite index of anthropometric failure (CIAF). Indian J Community Med. 2007;1 (1):35-9.

17. Shit S, Taraphdar P, Mukhopadhyay DK, Sinhababu A, Biswas AB. Assessment of nutritional status by composite index for anthropometric failure: a study among slum children in Bankura, West Bengal. Indian J Public Health. 2012;56:305-7.

18. International Institute for Population Sciences (IIPS) and Macro International. National Family Health Survey (NFHS-3), India, 2005-06: Maharashtra. Mumbai: IIPS; 2008.

19. Fidanza F, Keller W. Anthropometric Methodology. In: Fidanza F, editor. Nutritional Status Assessment - A Manual for Population Studies. London: Champman \& Hall; 1991. p. 6-11.

20. Heymsfield S. Anthropometric methodology. In: Fidanza F, editor. Nutritional status assessment - A manual for population studies. London: Chapman \& Hall; 1991. p. 24-8.

21. World Health Organization. The WHO Child Growth Standards. WHO website. http://www.who.int/childgrowth/standards/en/ Accessed on July 4, 2013

22. Deshmukh PR, Dongre AR, Sinha N, Garg BS. Acute childhood morbidities in rural Wardha: some epidemiological correlates and health care seeking. Indian J Med Sci. 2009;63:345-54.

23. Anjum F, Pandit MI, Mir AA, Bhat IA. Z score and CIAF - A comprehensive measure of magnitude of under nutrition in a rural school going population of Kashmir, India. Glob J Med Public Health. 2012;1(5):46-9.

\section{Submit your next manuscript to BioMed Central and take full advantage of:}

- Convenient online submission

- Thorough peer review

- No space constraints or color figure charges

- Immediate publication on acceptance

- Inclusion in PubMed, CAS, Scopus and Google Scholar

- Research which is freely available for redistribution 